\title{
A comparative study of chemical and immunological method of fecal occult blood test in the diagnosis of occult lower gastrointestinal bleeding
}

\author{
Yeasmin $\mathrm{F}^{1}$, Ali MA², Rahman $\mathrm{MA}^{3}$, Sultana $\mathrm{T}^{4}$, Rahman $\mathrm{MQ}^{4}$, Ahmed $\mathrm{ANN}^{4}$ \\ ${ }^{1}$ Department of Pathology, Ad-din Women's Medical College, Dhaka, ${ }^{2}$ Department of Hepatology Ad-din \\ Women's Medical College, Dhaka, ${ }^{3}$ Department of Gastroenterology, Bangabandhu Sheikh Mujib Medical \\ University (BSMMU), Dhaka, ${ }^{4}$ Department of Clinical Pathology, BSMMU, Dhaka. \\ Email: drnazmayasmeen@yahoo.com
}

\begin{abstract}
Fecal occult blood test is the most widely used screening test for diagnosis of gastrointestinal bleeding disorders specially colorectal carcinoma. Among the various methods of fecal occult blood tests, chemical method is being used commonly, but the method has some drawbacks like low participation rate, high false positive rate, low sensitivity etc. To overcome these short comings, newer immunological method was introduced. This study evaluated the role of immunological method of fecal blood test in the diagnosis of occult lower GIT bleeding. Stool samples from two hundred patients were examined by both chemical and immunological method. The patients who were positive by any or both methods of occult blood test, were advised for colonoscopy. During colonoscopy tissues were taken for histopathology which was the gold standard of this study. Among 110 OBT positive patients pathological lesions were detected in 65 patients by colonoscopy and histopathology. The diseases detected by colonoscopy and histopathology 18 colorectal polyp, 8 colorectal cancer, 24 ulcerative lesions and 5 inflammatory bowel disease etc. Regarding comparative analysis of chemical and immunological method, the higher sensitivity (95.4\% vs. $49.2 \%)$, specificity $(44.4 \%$ vs. $37.8 \%)$, accuracy $(74.5 \%$ vs. $44.5 \%)$, PPV (71.3\% vs. $53.3 \%)$ and NPV (87\% vs. $34 \%)$ of immunological method than chemical method was observed. Thus immunological method of fecal occult blood test was appeared to be a better alternative to conventional chemical method of fecal occult blood test in the diagnosis of occult lower GIT bleeding.
\end{abstract}

\section{Introduction}

Gastro intestinal bleeding disorders specially colorectal diseases are major public health issue worldwide. Occult gastrointestinal bleeding is the most common form of gastrointestinal bleeding and generally presents as iron deficiency anemia or presence of occult blood in stool ${ }^{1}$. The diseases causing occult blood in stools include intestinal polyps $(>1 \mathrm{~cm})$, diverticular bleeding, ischemic colitis, anal fissure, internal hemorrhoids, infections such as amoebiasis, ascariasis, hookworm, tuberculosis, colon cancer, lymphoma, inflammatory bowel diseases etc ${ }^{2}$. Among these conditions, colorectal cancer is the most important because of high incidence and mortality rate ${ }^{3}$. The methods commonly used to diagnose GIT bleeding disorders are colonoscopy, flexible sigmoidoscopy, CT colonography and virtual colonoscopy, barium enema, fecal occult blood test and detection of human DNA in stool ${ }^{4}$. Among these tests colonoscopy remains the gold standard for visualization, biopsy and removal of colonic polyps 5 . But it requires expertise and is expensive, carries risks and uncomfortable for the patients, some times require full anesthesia or intravenous medicine for patient's comfort ${ }^{6}$. The simplest and most evaluated screening methods available for detection of occult GIT bleeding is the fecal occult blood test. There are several methods for identification of occult blood in stool. Basically these are of three categories ${ }^{7}$. Guaiac-type test require the peroxidase like activity of the intact heam moiety. Immunological test target antigenic sequence on the globin chains of hemoglobin and finally, heme-prophyrin test are flurometric assay that include both heme and heme derived porphyrin in their measurement ${ }^{8}$. In most of the countries the guaiac based method is used?. But the test needs dietary restrictions, produce false positive and negative results, hence it has less reliability. On the other hand, immunological test require no dietary restrictions, highly sensitive and specific for human blood and single sample examination is sufficient. So far our knowledge, there is no study in Bangladesh regarding comparative study of various methods for diagnosis of lower GIT bleeding by 
different type of fecal occult blood test with colonoscopy and histologic evaluation. More over the prevalence of these diseases is not known to us. The aim of this study is to detect the better method of fecal occult blood test for early diagnosis of occult lower GIT bleeding by comparing the chemical and immunological method. Thus to reduce the incidence and mortality from GIT bleeding disorders by early diagnosis and further management of the patient.

\section{Materials and Methods}

This cross sectional study was carried out in the department of Clinical Pathology, BSMMU, Dhaka during the period October, 2008 to August, 2009. For this purpose a total number of 200 patients suspected to have occult bleeding were included from inpatient and out patient department of Gastroenterology and Colorectal surgery, BSMMU. Referred cases from other areas were also included. A total of 200 patients age ranging from 16 to 84 years of both sexes, with a male to female ratio of $2: 1$, were enrolled in the study according to inclusion criteria. Stool samples of all patients were examined by both chemical and immunological methods. According to presence / absence of occult blood in stool samples 120 cases having occult bleeding were considered as group I and 80 cases having no occult bleeding were considered as group II. The patients who were OBT positive by any one or both methods were advised for colonoscopy. As the study was to compare the effectiveness of chemical and immunological method of fecal occult blood test, Group II patients were not undergone colonoscopy. They were advised for regular follow up. Among 120 OBT positive patients, 110 patients underwent colonoscopy. Diseases detected by colonoscopy in group I patients, $18(16.4 \%)$ colorectal polyp, 24(21.8\%) colonic ulcer, $8(7.3 \%)$ colorectal cancer, $5(3.5 \%)$ inflammatory bowel disease, $7(6.4 \%)$ haemorrhoids and fissure, 2(1.8\%) colonic diverticulum and $1(0.9 \%)$ proctitis. Tissue was taken from these lesions and histopathological examination done.

\section{Results}

A total of 190 patients were finally included in the study, out of which 110 patients were OBT positive which was considered as group I and 80 patients who were OBT negative were considered as group II. The mean age was $36.7 \pm 14.6( \pm \mathrm{SD})$ years with their age ranged from 16 to 86 years in group I and while mean age in group II was $44.8 \pm 11.9( \pm \mathrm{SD})$ years with their age ranged from 22 to 78 years.
Maximum number of patients was found in age group 21-30 years in group I. Regarding the family history, it was observed that in group I, 38(34.5\%) and in group II, $15(18.8 \%)$ cases had positive family history of colorectal disease. Regarding the rate of participation in Guaiac method (GFOBT) it was found that one sample was collected from all patients in both groups. Two samples were collected from $22(20.0 \%)$ and $24(30.0 \%)$ in group I and group II respectively. Three samples were collected from $5(4.5 \%)$ in group I and $2(2.5 \%)$ in group II. Patients were also evaluated by immunological method (IFOBT) and found positive $87(79.1 \%)$ cases and negative in $23(20.9 \%$ ) cases in group I patients. According to the Colonoscopy findings it was observed that $45(40.9 \%)$ cases were normal, 18(16.4\%) found colonic polyp, 24(21.8\%) colonic ulcer, $3(2.7 \%)$ ulcerative colitis, $7(6.4 \%)$ haemorrhoid and fissure, $8(7.3 \%)$ colorectal cancer, 2(1.8\%) Crohn's disease, $2(1.8 \%)$ colonic diverticulum and $1(0.9 \%)$ proctitis in group I patients. The patients suspected to have occult bleeding were evaluated by G-FOBT and then compared with colonoscopy. Out of the 110 cases $60(54.5 \%)$ cases were positive and 50(45.5\%) cases were negative in G-FOBT. Among the 60 cases, which were OBT positive evaluated by G-FOBT, 32 cases were found disease positive and 28 cases were found disease negative in colonoscopy. Whereas, 33 cases were found disease positive and 17 cases were disease negative in colonoscopy among the negative cases, which were diagnosed by G-FOBT. Therefore, 65(59.1\%) cases were OBT positive and $45(40.9 \%)$ cases were OBT negative in colonoscopy. The difference was not statistically significant $(\mathrm{p}>0.05)$. $p$ value of 0.178 reached from chi square test and chi value was 1.81 with 1 degree of freedom. The patients were also evaluated by I-FOBT and then compared with colonoscopy. Out of the 110 cases $87(79.1 \%$ ) cases were positive and $23(20.9 \%)$ cases were negative in I-FOBT. Among the 87 cases, which were positive evaluated by I-FOBT, 62 cases were found disease positive and 25 cases were found disease negative in colonoscopy. Whereas, 3 cases were found disease positive and 20 cases were disease negative in colonoscopy among the negative cases, which were diagnosed by I-FOBT. Therefore, 65(59.1\%) cases were OBT positive and $45(40.9 \%$ ) cases were OBT negative in colonoscopy. The difference was statistically significant $(\mathrm{p}<0.05), \mathrm{p}$ value of 0.001 reached from chi square test and chi value was 25.51 with 1 degree of freedom.

In the present study we found the higher sensitivity of IFOBT for detection of colonic cancer, colorectal polyp and ulcerative lesions in the colon, $87.5 \%, 100 \%$ and $93.1 \%$ respectively. On the other 
hand the sensitivity of GFOBT for detection of colorectal cancer, colonic polyp, and ulcerative lesions were $75 \%, 38.9 \%$ and $51.7 \%$ respectively.

The specificity of IFOBT for detecting colorectal cancer, colonic polyp, and ulcerative lesions were $47.1 \%, 42.4 \%$ and $44.4 \%$ respectively. On the other hand the specificity of GFOBT for colorectal cancer, colonic polyp, and ulcerative lesions were $21.6 \%, 25 \%$ and $25.9 \%$ respectively.

PPV and of GFOBT were $8 \%, 11.7 \%$ and $25 \%$ for detection of colorectal cancer, polyps and ulcerative lesions respectively. While the PPV of IFOBT were $10 \%, 20.7 \%$ and $31 \%$ for detection of colorectal cancer, polyps and ulcerative lesions respectively. The NPV of GFOBT were $95.7 \%$, $78 \%$ and $72 \%$ for detection of colorectal cancer, polyps and ulcerative lesions. On the other hand the NPV of IFOBT were $96 \%, 100 \%$ and $91.3 \%$ for detection of colorectal cancer, polyps and ulcerative lesions respectively.

Table I: Rate of participation in G-FOBT and I-FOBT of the study subjects $(n=190)$

\begin{tabular}{lcccc}
\hline Rate of participation & \multicolumn{2}{c}{ Group I $(\mathrm{n}=110)$} & \multicolumn{2}{c}{ Group II $(\mathrm{n}=80)$} \\
\cline { 2 - 5 } & $\mathrm{n}$ & $\%$ & $\mathrm{n}$ & $\%$ \\
\hline G-FOBT & 110 & 100 & 80 & 100 \\
One sample & 22 & 20.0 & 24 & 30.0 \\
Two sample & 5 & 4.5 & 2 & 2.5 \\
$\quad$ Three sample & 110 & 100 & 80 & 100 \\
\hline Total & 87 & 79.1 & 0 & 0.0 \\
\hline I-FOBT (one sample) & 23 & 20.9 & 77 & 100 \\
$\quad$ Positive & 110 & 100 & 77 & 100 \\
$\quad$ Negative & \multicolumn{3}{c}{}
\end{tabular}

Table II: Comparison between G-FOBT and I-FOBT for identification of any pathology in colonoscopy $(n=110)$.

\begin{tabular}{lllc}
\hline & & \multicolumn{2}{c}{ Colonoscopy } \\
\hline \multirow{3}{*}{ G-FOBT } & Pathological & Normal \\
\cline { 2 - 2 } & Positive $(\mathrm{n}=60)$ & 32 & 28 \\
& Negative $(\mathrm{n}=50)$ & 33 & 17 \\
\cline { 2 - 4 } & Total $(\mathrm{n}=110)$ & 65 & 45 \\
\hline \multirow{3}{*}{ I-FOBT } & Positive $(\mathrm{n}=87)$ & 62 & 25 \\
\cline { 2 - 4 } & Negative $(\mathrm{n}=23)$ & 3 & 20 \\
\cline { 2 - 4 } & Total $(\mathrm{n}=110)$ & 65 & 45 \\
\hline
\end{tabular}

Chi value $=1.81, \mathrm{p}$ value $=0.178$ degree of freedom $(\mathrm{df})=1(\mathrm{G}-\mathrm{FOBT})$ Chi value $=25.51$, $p$ value $=0.001$ degree of freedom $(\mathrm{df})=1(\mathrm{I}-\mathrm{FOBT})$

Table III: Sensitivity, specificity, accuracy and positiveand negative predictive values of the G-FOBT andI-FOBT for detection of occult bleeding $(\mathrm{n}=110)$.

\begin{tabular}{lcc}
\hline Validity test & G-FOBT $(\%)$ & I-FOBT $(\%)$ \\
\hline Sensitivity & 49.2 & 95.4 \\
Specificity & 37.8 & 44.4 \\
Accuracy & 44.5 & 74.5 \\
PPV & 53.3 & 71.3 \\
NPV & 34.0 & 87.0 \\
\hline
\end{tabular}

Table IV: Sensitivity, specificity, positive and negative predictive values of the G-FOBT and I-FOBT for identification of Colorectal cancer, Colonic polyp and ulcerative lesions $(n=110)$.

\begin{tabular}{lcccccc|}
\hline & \multicolumn{3}{c}{ G-FOBT(\%) } & \multicolumn{3}{c|}{ I-FOBT $(\%)$} \\
\hline Validity test & Cancer & Polyp & Ulcer & Cancer & Polyp & Ulcer \\
\hline Sensitivity & 75.0 & 38.9 & 51.7 & 87.5 & 100.0 & 93.1 \\
Specificity & 21.6 & 25.0 & 25.9 & 47.1 & 42.4 & 44.4 \\
PPV & 8.0 & 11.7 & 25.0 & 10.0 & 20.7 & 31.0 \\
NPV & 95.7 & 78.0 & 72.0 & 96.0 & 100.0 & 91.3 \\
\hline
\end{tabular}

\section{Discussion}

This study aimed to compare the usefulness of chemical and immunological method of fecal occult blood test in the diagnosis of occult lower GIT bleeding. Result from this study showed that immunological method has high participation rate and higher validity and reliability than chemical method.

Reduction in morbidity and mortality rates from disease causing occult bleeding by FOBT screening can be enhanced by high participation levels in the community. Choice of method is one factor that may influence participation. A simplified sampling process and reduction in number of required samples are associated with increases participation ${ }^{10}$. This may indicate a preference for immunological method which has no dietary restrictions and are often designed to be more user friendly ${ }^{11}$. Three randomized control trials of chemical method of fecal occult blood test (GFOBT) have found lower participation rate of $17 \%$, $22 \%$ and $33 \%$ respectively ${ }^{12}$. We also found the lower participation rate of GFOBT than IFOBT. One stool sample was collected from all the study subjects but two stool samples were collected from forty six patients and only seven patients were convinced to give three consecutive samples. This interpretation is consistent with the literatures which concluded that patient prefer the user friendly characteristics of immunological test ${ }^{13,14}$. A telephone survey found that $17 \%$ patients indicated that dietary restrictions discourage them from participation in chemical test and this proportion increased with age and was particularly related to medication restrictions.

The overall sensitivity, specificity, accuracy, PPV and NPV of IFOBT were $95.4 \%, 44.4 \%, 74.5 \%$, $71.3 \%$ and $87 \%$ respectively. On the other hand, the overall sensitivity, specificity, accuracy, PPV and NPV of GFOBT were $49.2 \%, 37 \%, 8 \%$, $44.5 \%, 53.3 \%$ \& $34 \%$ respectively. In a comparative study, Ranshoff ${ }^{15}$ found the sensitivity of IFOBT $95.9 \%$, and specificity $59.2 \%$, resulting in a 30\% reduction of colonoscopy use. This findings is very much consistent with our findings. A multicenter prospective case control study by 
Lohsiriwat $^{16}$ in Thailand found the overall sensitivity, specificity, positive predictive value, and negative predictive value of IFOBT were $91.0 \%, 93.8 \%, 95.8 \%$ \& $87.0 \%$ respectively, which supported the present study.

In this study we also calculated individually the accuracy of GFOBT and IFOBT for detection of colonic polyp, colorectal cancer and ulcerative lesions in the colon. As the incidence of colorectal cancer is more common in western countries, the comparative study between the two methods of OBT were done to detect colorectal cancer and colonic polyps in OBT positive patients. Although in developing country, the prevalence of colorectal cancer is low $(1 / 100,000)$, the incidence is increasing day by day due to change in life style, dietary habit and huge urbanization ${ }^{17}$. The relative risk of developing colorectal carcinoma in Bangladesh is also high ${ }^{\mathbf{1 8}}$. In this perspective, it is necessary to screen colorectal cancer among our asymptomatic average risk population of our country to reduce it's incidence and prevalence rate. Although polyps and ulcerative lesions are benign but it is proved that in time they may progress to invasive cancers. Polyps, specially larger $(>1 \mathrm{~cm})$ polyps become colorectal cancer at a rate of roughly $1 \%$ per year ${ }^{9}$. Considering this fact we had evaluated the individual performance characteristics of GFOBT and IFOBT for detection of polyp and cancer.

In the present study we found the higher sensitivity of IFOBT for detection of colonic polyp, colorectal cancer and ulcerative lesions in the colon, $87.5 \%$, $100 \%$ and $93.1 \%$ respectively. On the other hand, the sensitivity of GFOBT for detection of colorectal cancer, colonic polyp, and ulcerative lesions were $75 \%, 38.9 \%, 51.7 \%$ respectively. These findings are nearly similar to the findings noted by Simon ${ }^{19}$ where higher sensitivity of IFOBT (75\% to over $90 \%$ ) than GFOBT were found. On the other hand, sensitivity of GFOBT can be achieved as high as $90 \%$ by using repeated test, rehydration and diet restriction. This study recommended that the newer generation of test such as immunological test would undoubtedly proved even better in practice. Morikawa et al. ${ }^{\mathbf{2 0}}$ also found higher sensitivity of IFOBT in a study among 21805 Japanese population. Allison et al. ${ }^{\mathbf{9}}$ found that the sensitivity of IFOBT for detecting the colorectal cancer was $82 \%$, substantially higher than that of GFOBT. The estimated sensitivity of GFOBT for cancer ranged from $13 \%$ to $60 \%$ in the five economic models of colon cancer screening in average risk adults. Although there were differences in results (ranged from $66 \%$ to $98 \%$ ), the sensitivity of IFOBT in many published studies was much higher than results reported for GFOBT, which suggested that the method would show improved performance over GFOBT in screening programmes ${ }^{\mathbf{1 0}}$.

Regarding specificity, in this study we found higher specificity of IFOBT than GFOBT. The specificity of IFOBT for detecting colorectal cancer, colonic polyp, and ulcerative lesions were $47.1 \%, 42.4 \%$, $44.4 \%$ respectively. On the other hand, the specificity of GFOBT for colorectal cancer, colonic polyp, and ulcerative lesions were $21.6 \%, 25 \%$, $25.9 \%$ respectively. The higher specificity of IFOBT was also noted by Burch et al. $^{21}$ who reported the eight diagnostic case control studies regarding comparison of two methods. Among these 8 studies, IFOBT had higher specificities in four studies, another four studies founded comparable specificity of both methods.

In the present study we had analyzed positive and negative predictive value of GFOBT and IFOBT for detection of colorectal cancer, polyps and ulcerative lesions. It was observed that PPV and of GFOBT were $8 \%, 11.7 \%$ and $25 \%$ for detection of colorectal cancer, polyps and ulcerative lesions respectively. Again the PPV of IFOBT were $10 \%$, $20.7 \%$ and $31 \%$ for detection of colorectal cancer, polyps and ulcerative lesions respectively. The NPV of GFOBT were $95.7 \%, 78 \%$ and $72 \%$ for detection of colorectal cancer, polyps and ulcerative lesions. On the other hand the NPV of IFOBT were $96 \%, 100 \%$ and $91.3 \%$ for detection of colorectal cancer, polyps and ulcerative lesions respectively. Guittet et al. ${ }^{\mathbf{2 2}}$ also observed higher(8.7\% vs $7.3 \%$ ) PPV of IFOBT than GFOBT for detection of polyp and (49.2\% vs $27.2 \%)$ for detection of colorectal neoplasia.

The potential usefulness of IFOBT compared with GFOBT was being considered by several organizations. The American Cancer Society's recommended that in comparison with guaiacbased tests for the detection of occult blood, immunological tests were more patient friendly and were likely to be equal or better in sensitivity and specificity. The World Health Organization and the World Organizaton for Digestive Endoscopy have also endorsed the use of immunological test because population cannot be relied on to comply with the dietary and drug restrictions necessary for guaiac- based tests ${ }^{\mathbf{2 3}}$.

Evidence in favour of the use of IFOBT over the GFOBT is increasing. A number of variables may influence guaiac test results that do not influence the results of immunological tests. Because guaiac tests depend upon peroxidase or pseudoperoxidase activity in feaces not specific to human hemoglobin. So the ingestion of animal 
hemoglobin/ myoglobin in red meat, fruits and vegetables high in peroxidase activity, and aspirin and other medication that may produce gastrointestinal bleeding, may cause false-positive results and high dose of vitamin $\mathrm{C}$ and fecal dehydration which may cause false negative results. IFOBT have no dietary or medication restrictions. These tests have superior sensitivity and specificity and a higher compliance rate as suggested in recent reviews.

Limitation of the study: There were some limitations in this study. First of all who were OBT negative by initial screening were not undergone for further evaluation. But there might be any disease among these average risk patients which could be evaluated and treated as well. However these patients were advised to visit their physician regularly. Secondly, exact incidence and prevalence of colorectal carcinoma could not be find out by this study because some suspected patients of colorectal cancer presented with visible per rectal bleeding which was an exclusion criteria. Indeed, our view of this study was to find out the better method of occult blood rest which can be used in large scale screening programme. Finally, patients could not take necessary medication during three days preparatory period for fecal occult blood test.

\section{Acknowledgement}

I would like to thank all the consultants, junior doctors and staff of the Department of Clinical Pathology, BSMMU, Dhaka for their sincere help in conducting this study.

\section{References}

1. Beg M, Shing M, Saraswat MK, Rewan BB. Occult Gastrointestinal Bleeding: Detection, Interpretation and Evaluation. JIACM.2002; 3(2):153-8.

2. Zuckerman GR. Acute gastrointestinal bleeding: Clinical essentials for the initial evaluation and risk assessment. Gastroenterol.2000; 118(1):201-2.

3. Tasende JD, Gabriel JC. Colorectal cancer screening with fecal occult blood testing. REV ESP ENFERM DIG (Madrid).2008; 100( 6):315-319.

4. Levin B,Brooks D, Smith RA, Stone A.Emerging Technologies in Screening for Colorectal Cancer :CT Colonography, Immunochemical Fecal Occult Blood Tests, and Stool Screening Using Molecular Markers. CA Cancer J Clin.2003; 53:44-55.

5. Donald E, Mansell M. Colon polyps and Colon cancer. Retrieved on June 4,2009 from http://personalweb. sunset.net/ mansell/polyp.htm.

6. Palmer KR, Penman ID and Paterson-Brown S.Alimentary tract and pancreatic disease, in Davidson's Principles and Practice of Medicine.2006. $20^{\text {th }}$ edn, Elsevier, New Delhi, 912-920.

7. Ahmed ANN. Faecal occult blood test (FOBT)- Recent Concept. Bangladesh J of Pathol.1999;14( 2):1-2.
8. Mitchell SH, Schaefer DC \& Dubagunta S.A new view of occult \& obscure gastrointestinal bleeding. J of American Academy of Family Physicians. 2004;69:87581.

9. Allison JE, Sakoda LC, Levin TR, Tucker JP.Screening for Colorectal Neoplasms With New Fecal Occult Blood Tests: Update on Performance Characteristics. Journal of the National Cancer Institute. 2007; 99(19): 1462-1470.

10. Cole SR, Young GP, Esterman A, Cadd B, Morcom J.A randomized trial of the impact of new faecal hemoglobin test technologies on population participation in screening for colorectal cancer.J Med Science.2003;10:117-22.

11. Smith, A, Young, G.Comparison of a brush-sampling fecal immunochemical test for hemoglobin with a sensitive guaiac-based occult blood test in detection of colorectal neoplasia. Cancer.2006;107(9):2152-59.

12. Allison JE, Lawson, M. Screening Tests for Colorectal Cancer. Current Oncology Reports. 2006; 8: 492-498.

13. Hundt S, Haug $U$ and Brenner H. Comparative Evaluation ofImmunochemical Fecal Occult Blood Tests for Colorectal Adenoma Detection. Annals of Internal Medicine. 2009;150:162-169.

14. James E Allison, MD, Irene S. Tekawa, M.A., Laura J. Ransom, M.S., and Alyn L. Adrain, M.A Comparison of Fecal Occult blood tests for colorectal cancer screening. NEJM. 2008; 334(3 ):155-160.

15. Ranshoff $\mathrm{D}$. What is the role of iFOBT in screening for colorectal cancer.Gut.2007; 56:1343-1344.

16. Lohsiriwat $\mathrm{V}$, Thavichaigarn $\mathrm{P}$, Awapittaya BA. Multicenter Prospective Study of Immunochemical Fecal Occult Blood Testing for Colorectal Cancer Detection. J Med Assoc Thai. 2007; 90(11) :2291-5.

17. Scanga A. Obesity \& colorectal cancer [ppt].2007. Retrieved Dec. 16, 2008 from http://www.uwgi. org/ calendar/presentations/2007_2008/20070824_scanga.p df

18. Kumar V, Abbas AK, Fausto N 2004, Neoplasia, Robin`s and Cotran Pathologic basis of diseases, $5^{\text {th }}$ edition. W.B. Saunders company, Philadelphia, pp.783-800.

19. Simon JB. The pros and cons of fecal occult blood testing for colorectal neoplasms. Cancer and Metastasis Reviews.1987; 6:397-411.

20. Morikawa T, Kato J,Yamaji Y, Wada R, Mitsushima T. A Comparison of the immunochemical fecal occult blood test and total colonoscopy in the asymptomatic population. Gastroenterol, 2005;129:422-428.

21. Burch JA, Weiser KS,John DJ, Duffy S,Smith S.Diagnostic accuracy of faecal occult blood tests used in screening for colorectal cancer: a systematic review.J of medical screening. 2007;14( 3):132-137.

22. Guittet L, Bouvier V, Mariotte N, Vallee J P, Arsène D, Boutreux S, Tichet J, Launoy G. Comparison of a guaiac based and an immunochemical faecal occult blood test in screening for colorectal cancer in a general average risk population.Gut.2007;56:210-214.

23. Hughes K, Legget B, Delmar C, Aitken J. Guaiac versus immunochemical tests: fecal occult blood test screening for colorectal cancer in a rural community. Aust and NZ J of public health, 2005; 29(4):358-364. 